\title{
HER2-positive recurrent breast cancer and metastases of breast cancer, including life-threatening metastases to the brain and dura mater: a case effectively treated with lapatinib plus capecitabine before whole brain radiotherapy
}

\author{
Kazuo Ishizuna - Jun Ninomiya - Makoto Kojima - Erina Nakane • \\ Fumiko Nishibayashi $\cdot$ Miho Kawashima Miwako Nozaki • \\ Hidetsugu Yamagishi $\cdot$ Yoshihiko Ueda $\cdot$ Masatoshi Oya
}

Received: 23 June 2012/ Accepted: 17 August 2012/Published online: 29 September 2012

(C) The Japan Society of Clinical Oncology 2012

\begin{abstract}
Lapatinib is a new small molecule tyrosine kinase inhibitor. It targets both ErbB1 and ErbB2 (HER2) receptors and directly and reversibly acts on them to inhibit signaling. Since lapatinib has a low molecular weight, it can cross the blood-brain barrier. Thus, there is much interest in its potential effectiveness against brain metastases. Our patient was a 57-year-old postmenopausal woman with a non-contributory medical history. In September 2006, she underwent a partial mastectomy and axillary dissection for right breast cancer. Pathological diagnosis was solid-tubular carcinoma, $n+(6 / 23)$, nuclear grade 3, ly1, v0, ER+, PgR-, and HER2 3+ (pT1cN2a M0-stageIIIA). Adjuvant chemotherapy consisted of 6 cycles of epirubicin and cyclophosphamide (EC) and subsequent oral anastrozole. In May 2009, the patient developed choroidal, lung, liver, bone, and lymph node metastases. She underwent radiation therapy (choroids 40 Gy and cervical vertebrae $40 \mathrm{~Gy}$ ), and the oral drug was changed to high-dose toremifene. In July 2009, the patient
\end{abstract}

K. Ishizuna $(\bowtie) \cdot$ M. Kojima $\cdot$ E. Nakane $\cdot$ M. Oya

Breast Center, Dokkyo Medical University Koshigaya Hospital,

2-1-50 Minami-Koshigaya, Koshigaya, Saitama 343-8555, Japan

e-mail: k.ishizuna@hotmail.co.jp

J. Ninomiya

Ninomiya Hospital, 491-6 Shin-eicho, Soka,

Saitama 340-0056, Japan

F. Nishibayashi $\cdot$ M. Kawashima $\cdot$ M. Nozaki

Department of Radiology, Dokkyo Medical University

Koshigaya Hospital, 2-1-50 Minami-Koshigaya, Koshigaya,

Saitama 343-8555, Japan

H. Yamagishi · Y. Ueda

Department of Pathology, Dokkyo Medical University

Koshigaya Hospital, 2-1-50 Minami-Koshigaya, Saitama,

Koshigaya 343-8555, Japan developed headache, vomiting, and seizures. MRI revealed multiple brain metastases and dura mater metastases. Administration of glycerol and steroid was begun. In addition, administration of lapatinib $(1,250 \mathrm{mg} / \mathrm{body}$, daily) plus capecitabine $\left(2,000 \mathrm{mg} / \mathrm{m}^{2}\right.$, day $1-14$, every 3 weeks) was begun for treatment of brain and systemic metastatic lesions. Neurological symptoms improved on day 14. In January 2010, examinations revealed partial response of all metastatic lesions, including brain metastases. The patients continued the same treatment but exhibited progressive disease in March 2010. Thus, the treatment was discontinued as per the patient's request. In May 2010, the patient developed generalized seizures again and underwent whole brain radiotherapy (30 Gy). However, the patient's general condition gradually deteriorated and the patient died in June 2010.

Keywords Breast cancer - Brain metastasis .

Lapatinib · Capecitabine

\section{Introduction}

It is often difficult to cure metastatic breast cancer and recurrent breast cancer, except for some local recurrences. Approximately $10-16 \%$ of the patients with metastatic breast cancer or recurrent breast cancer have brain metastases, and such patients have poor prognosis [1]. The effectiveness of pharmacotherapy is not high in the brain because of the blood-brain barrier. Therefore, the standard treatment of brain metastasis is radiation therapy. However, the molecularly targeted drug lapatinib has a low molecular weight and can cross the blood-brain barrier. Thus, there is much interest in its potential effectiveness against brain metastases [2]. 
Fig. 1 In July 2009, MRI revealed multiple brain metastases with marked cerebral edema and dura mater metastases. CT revealed lung, liver, and bone metastases (a, b head MRI; c, d contrastenhanced CT)
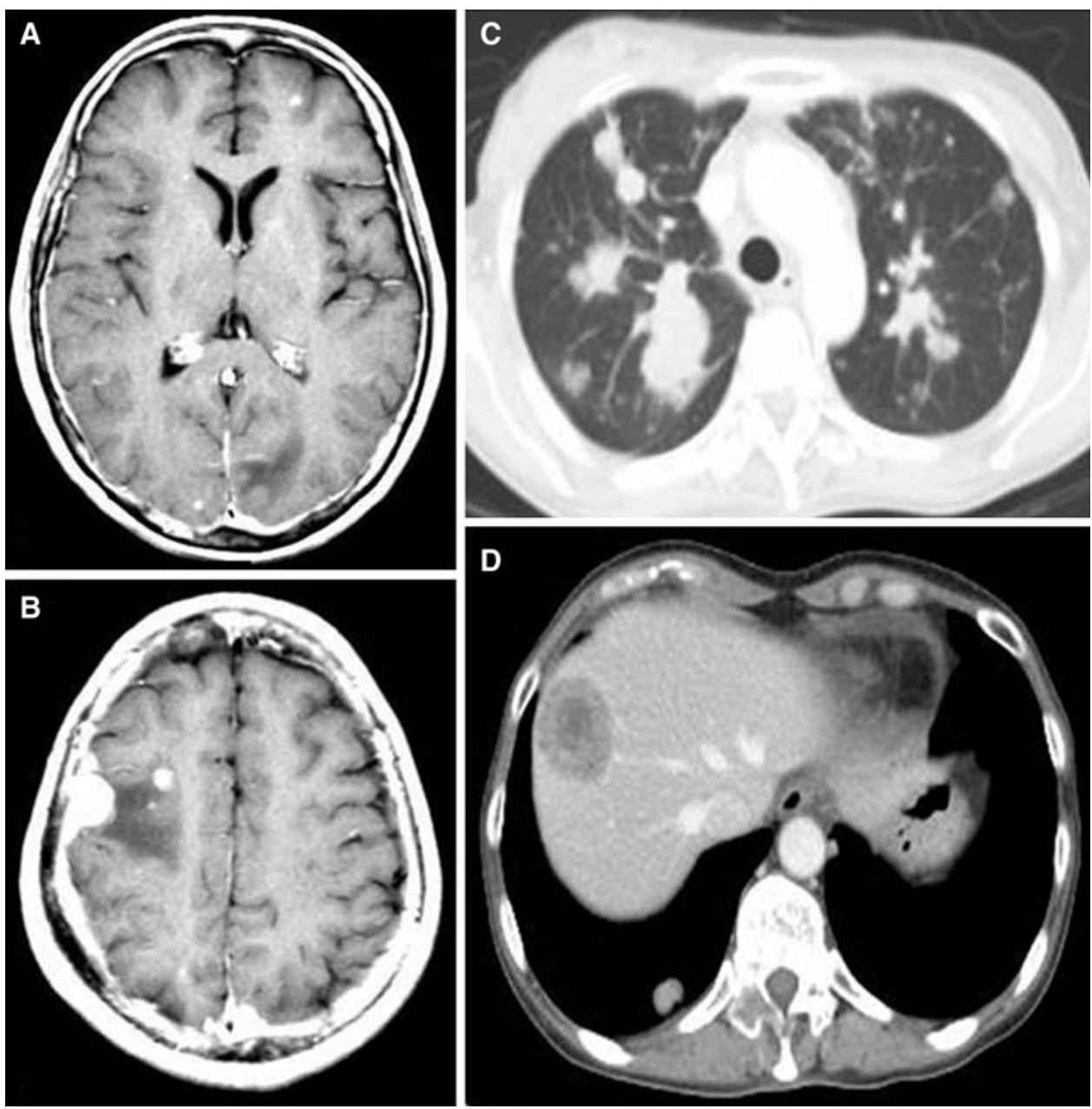

In this report, we present a case of HER2-positive recurrent breast cancer and metastases of breast cancer, including life-threatening metastases to the brain and dura mater. The patient was effectively treated using lapatinib plus capecitabine before whole brain radiotherapy. We also include a brief literature review.

\section{Case report}

The patient was a 57-year-old postmenopausal woman with a non-contributory medical history. In September 2006, she underwent a partial mastectomy and axillary dissection for right breast cancer (T2N1M0, stage IIB). Pathological diagnosis was solid-tubular carcinoma, $n+(6 / 23)$, nuclear grade 3 , ly1, v0, estrogen receptor (ER) positive, progesterone receptor $(\mathrm{PgR})$ negative, and HER2 $3+[\mathrm{pT} 1 \mathrm{cN} 2 \mathrm{a}$ M0-stageIIIA (UICC)]. Adjuvant chemotherapy consisted of 6 cycles of epirubicin and cyclophosphamide (EC 90 and $600 \mathrm{mg} / \mathrm{m}^{2}$, respectively, every 3 weeks) and subsequent oral anastrozole (1 mg/day). In May 2009, the patient developed reduced visual acuity and underwent examinations which revealed choroidal, lung, liver, bone, and lymph node metastases. She underwent radiation therapy (choroids $40 \mathrm{~Gy}$ and cervical vertebrae $40 \mathrm{~Gy}$ ), and the oral drug was changed to high-dose toremifene (120 mg/day). In July 2009, the patient developed headache, vomiting, and seizures and was admitted to our hospital. MRI revealed multiple brain metastases and dura mater metastases, and CT revealed enlarged lung, liver, and bone metastases (tumor markers: CEA $=6.8 \mathrm{ng} / \mathrm{ml}$ and CA15-3 = 44.4 U/ml) (Fig. 1a-d). Since the metastases were life-threatening, we recommended trastuzumab plus weekly paclitaxel and whole brain radiotherapy. The patient did not consent to intravenous chemotherapy because she experienced severe nausea with EC administration. She also had strong concerns regarding whole brain radiotherapy and did not accept the aforementioned treatment. The patient underwent intravenous administration of glycerol and steroid for cerebral edema. She consented to oral chemotherapy consisting of lapatinib and capecitabine. In August 2009, administration of lapatinib (1,250 mg/ body, daily) plus capecitabine $\left(2,000 \mathrm{mg} / \mathrm{m}^{2}\right.$, day $1-14$, every 3 weeks) was begun for treatment of brain and 
Fig. 2 In January 2010 (after completion of 7 cycles of lapatinib plus capecitabine), there was partial response of all metastatic lesions, and the tumor volume reduction rate was $31 \%$ for brain metastases and $65 \%$ for dura mater metastases (a, b head MRI; c, d contrast-enhanced CT)
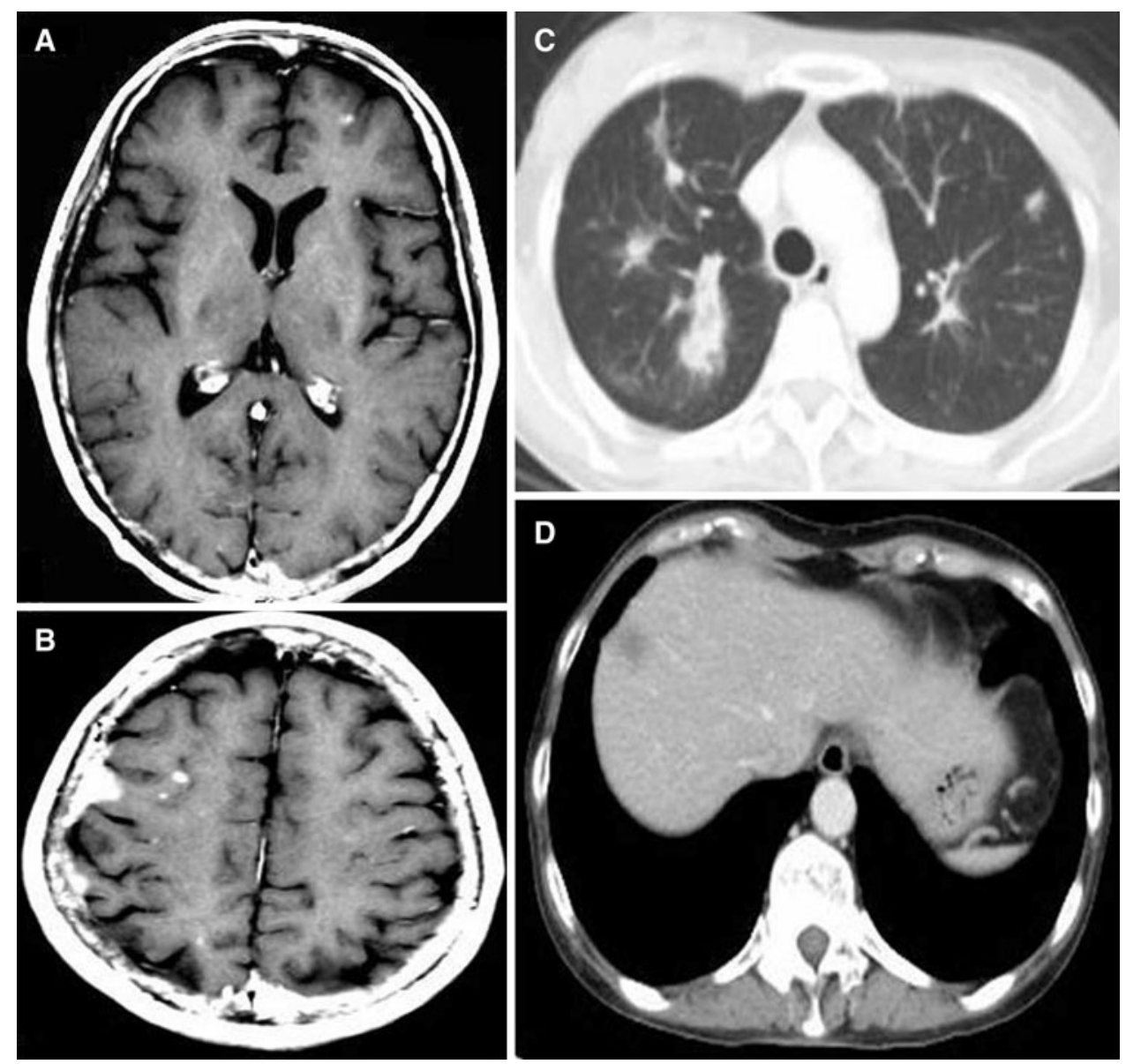

systemic metastatic lesions. Neurological symptoms improved on day 14. Administration of glycerol and steroid was discontinued, and the patient was discharged. In January 2010 (after completion of 7 cycles), examinations revealed that systemic, brain, and dura mater metastases had reduced in size, indicating a partial response (tumor markers: $\quad$ CEA $=3.5 \mathrm{ng} / \mathrm{ml}, \quad$ CA15-3 $=24.9 \mathrm{U} / \mathrm{ml}$ ) (Fig. 2a-d). There were no side effects. In March 2010 (after completion of 10 cycles), the patient exhibited progressive disease. Thus, the treatment was discontinued as per the patient's request (tumor markers: CEA $=5.6 \mathrm{ng}$ / $\mathrm{ml}, \mathrm{CA} 15-3=50.1 \mathrm{U} / \mathrm{ml}$ ). In May 2010, the patient developed generalized seizures again and underwent whole brain radiotherapy ( $30 \mathrm{~Gy}$ ). However, the patient did not improve and died in June 2010.

\section{Discussion}

Approximately $20-30 \%$ of breast cancer cases show HER2 protein overexpression [3, 4], and the prognosis of these patients has greatly improved owing to the development of trastuzumab [5, 6]. HER2-positive breast cancer is known to frequently metastasize to the brain [7, 8]. High effectiveness of trastuzumab cannot be expected for brain metastases because of its large molecular weight and inability to cross the blood-brain barrier $[9,10]$. Thus, it is important to find an effective treatment to control brain metastatic lesions.

Lapatinib is a new small molecule tyrosine kinase inhibitor that targets both ErbB1 and ErbB2 (HER2) receptors. It directly and reversibly binds to the ATPbinding site of the kinase in the intracellular domain, resulting in the inhibition of ErbB1 and ErbB2 autophosphorylation and inhibition of signaling [11].

Cameron et al. [12] conducted a randomized phase III trial involving patients with HER2-positive metastatic breast cancer or recurrent breast cancer that was resistant to trastuzumab. These patients were treated with capecitabine monotherapy or lapatinib plus capecitabine. Lapatinib plus capecitabine was shown to be more effective than capecitabine monotherapy (median progression-free survival 6.2 vs. 4.3 months, respectively; hazard ratio $0.57,95 \%$ CI $0.43-0.77, p<0.001$ ) [12]. Lapatinib plus capecitabine is considered to be a promising treatment for HER2-positive metastatic breast cancer and recurrent breast cancer resistant to trastuzumab. 
Since lapatinib has a low molecular weight, it can pass through the blood-brain barrier. Thus, there is much interest in its potential effectiveness against brain metastases [2]. Several trials have shown that lapatinib was effective against HER2-positive breast cancer brain metastases.

Lin et al. [13] conducted a phase II trial involving subjects who had HER2-positive metastatic breast cancer to the brain that was resistant to trastuzumab and who had undergone whole brain radiotherapy. Lapatinib monotherapy was used in 236 patients, of whom 19 patients ( $8 \%$ ) had a tumor volume reduction of $50 \%$ or more, and 50 patients $(21 \%)$ had a volume reduction of $20 \%$ or more. A combination of lapatinib and capecitabine was used in 50 patients, of whom 11 patients (22\%) had a volume reduction of $50 \%$ or more, and 20 patients (40\%) had a volume reduction of $20 \%$ or more [13].

Metro et al. [14] examined patients who had HER2positive metastatic breast cancer to the brain after whole brain radiotherapy and who were treated with trastuzumab. The study compared patients who continued to use trastuzumab and those who switched to lapatinib plus capecitabine. Lapatinib plus capecitabine significantly prolonged the mean survival compared with trastuzumab (16.7 vs. 27.9 months, $p=0.01$ ) [14].

Radiation therapy is the standard treatment of breast cancer brain metastasis. There has also been a trial regarding lapatinib plus capecitabine used up front. Bachelot et al. [15] conducted a phase II trial involving patients with HER2-positive metastatic breast cancer to the brain. These patients received lapatinib plus capecitabine before whole brain radiotherapy. The results showed that, of 43 patients, 29 (67.4\%, $95 \%$ CI 51-81) achieved an objective central nervous system response (CNS-OR), which was defined as a $50 \%$ or more volume reduction of CNS lesions. Time to progression was 5.5 months (95\% CI 3.9-5.9). There were 32 patients (74.4\%) who had undergone whole brain radiotherapy owing to progression of CNS lesions. The median time until whole brain radiotherapy was 8.3 months (95\% CI 5.1-11.7), and the 6-month survival rate was $90.9 \%$ (95\% CI 77.4-96.5). The above results suggest that lapatinib plus capecitabine can achieve high response rates in patients with HER2positive breast cancer who were newly diagnosed with brain metastases, and that lapatinib plus capecitabine is a promising new treatment option for such patients [15].

In our case, the patient had severe side effects of intravenous adjuvant chemotherapy. Thus, the patient did not consent to the use of trastuzumab. Since lapatinib plus capecitabine is administered orally, patient acceptance is high. Our patient also accepted oral lapatinib plus capecitabine. Radiation therapy was not performed as per the patient's request, and lapatinib plus capecitabine was used for brain metastases and systemic metastases. The results indicated that the treatment was effective for these metastases. (In January 2010, MRI revealed a tumor volume reduction rate of $31 \%$ for brain metastases and $65 \%$ for dura mater metastases.) Neurological symptoms such as seizures, vomiting, and headache were controlled, and metastases were well controlled. Time to progression was 7.5 months, and good results were obtained for breast cancer brain metastases without radiation therapy. In addition, there were no side effects such as diarrhea or hand-foot syndrome.

It is often difficult to cure metastatic breast cancer and recurrent breast cancer. The prognosis of brain metastasis is particularly poor, and improvement of quality of life and extension of survival are the objectives of treatment for brain metastasis. Since lapatinib plus capecitabine is orally administered, patient acceptance is high and these drugs can become a treatment option for breast cancer brain metastasis.

\section{Conclusion}

In this report, we presented a case of HER2-positive recurrent breast cancer and metastases of breast cancer, including life-threatening metastases to the brain and dura mater. The patient was effectively treated using lapatinib plus capecitabine before whole brain radiotherapy. The result of our case showed that lapatinib plus capecitabine can be an effective treatment option for breast cancer brain metastases.

Conflict of interest The authors declare that they have no conflict of interest.

\section{References}

1. Lin NU, Bellon JR, Winer EP (2004) CNS metastases in breast cancer. J Clin Oncol 22:3608-3617

2. Gril B, Palmieri D, Bronder JL et al (2008) Effect of lapatinib on the outgrowth of metastatic breast cancer cells to the brain. J Natl Cancer Inst 100:1092-1103

3. Masood S, Bui MM (2000) Assessment of HER-2/neu overexpression in primary breast cancers and their metastatic lesions: an immunohistochemical study. Ann Clin Lab Sci 30:259-265

4. Slamon DJ, Clark GM, Wong SG et al (1987) Human breast cancer: correlation of relapse and survival with amplification of the HER-2/neu oncogene. Science 235:177-182

5. Marty M, Cognetti F, Maraninchi D et al (2005) Randomized phase II trial of the efficacy and safety of trastuzumab combined with docetaxel in patients with human epidermal growth factor receptor 2-positive metastatic breast cancer administered as first-line treatment: the M77001 study group. J Clin Oncol 23:4265-4274

6. Slamon DJ, Leyland-Jones B, Shak S et al (2001) Use of chemotherapy plus a monoclonal antibody against HER2 for metastatic breast cancer that overexpresses HER2. N Engl J Med 344:783-792 
7. Gobos Z, Sinha R, Hanson J et al (2006) Prognostic significance of human epidermal growth factor receptor positively for the development of brain metastasis after newly diagnosed breast cancer. J Clin Oncol 24:5658-5663

8. Pestalozzi BC, Zahrieh D, Price KN et al (2006) Identifying breast cancer patients at the risk for central nervous system metastases in trials of the international breast cancer study group. Ann Oncol 17:935-944

9. Pestalozzi BC, Brignoli S (2000) Trastuzumab in CSF. J Clin Oncol 18:2349-2351

10. Stemmler HJ, Schmitt M, Willems A et al (2007) Ratio of trastuzumab levels in serum and cerebrospinal fluid is altered in HER2-positive breast cancer patients with brain metastases and impairment of blood-brain barrier. Anticancer Drugs 18:23-28

11. Rusnak DW, Lackey K, Affleck K et al (2001) The effects of the novel, reversible epidermal growth factor receptor/ErbB-2 tyrosine kinase inhibitor, GW2016, on the growth of human normal and tumor-derived cell lines in vitro and in vivo. Mol Cancer Ther 1:85-94
12. Cameron D, Casey M, Press M et al (2008) A phase III randomized comparison of lapatinib plus capecitabine versus capecitabine alone in women with advanced breast cancer that has progressed on trastuzumab: updated efficacy and biomarker analyses. Breast Cancer Res Treat 112:533-543

13. Lin NU, Dieras V, Paul D et al (2009) Multicenter phase II study of lapatinib in patients with brain metastases from HER2-positive breast cancer. Clin Cancer Res 15:1452-1459

14. Metro G, Foglietta J, Russillo M et al (2011) Clinical outcome of patients with brain metastases from HER2-positive breast cancer treated with lapatinib and capecitabine. Ann Oncol 22:625-630

15. Bachelot TD, Romieu G, Campone $M$ et al (2011) LANDSCAPE: an FNCLCC phase II study with lapatinib (L) and capecitabine $(\mathrm{C})$ in patients with brain metastases (BM) from HER2-positive metastatic breast cancer (MBC) before wholebrain radiotherapy (WBR). J Clin Oncol (Meeting Abstracts) 29(15):509 